\title{
Knowledge-Aided Two-Dimensional Autofocus for Synthetic Aperture Radar
}

\author{
Xinhua Mao, Daiyin Zhu \\ Department of Electronic and Engineering \\ Nanjing University of Aeronautics and Astronautics \\ Nanjing, China \\ Email: \{xinhua, zhudy\}@nuaa.edu.cn
}

\author{
Yimin D. Zhang \\ Center for Advanced Communications \\ Villanova University \\ Villanova, PA 19085, USA \\ Email: yimin.zhang@villanova.edu
}

\begin{abstract}
Conventional two-dimensional (2-D) autofocus algorithms blindly estimate the phase error in the sense that they do not take advantages of the known structure of the phase error. As such, they do not achieve accurate estimation of the 2$D$ phase error due to lack of data redundancy. In this paper, we propose a novel 2-D autofocus algorithm that exploits the phase structure information, which is known a priori. The proposed method is described in two parts. We first reveal the analytical structure of the 2-D residual phase error in synthetic aperture radar (SAR) imageries under the polar format algorithm framework. Based on this result, the proposed 2-D autofocus approach converts the 2-D phase error estimation problem into a one-dimensional (1-D) problem of azimuth phase error estimation. Based on the phase structure, the 2-D phase error can then be computed from the estimated azimuth phase error. Experimental results clearly demonstrate the effectiveness of the proposed method.
\end{abstract}

\section{INTRODUCTION}

Synthetic aperture radar (SAR) is a coherent imaging system which, by coherently processing multiple echo pulses, provides high azimuth resolution. Coherent data processing requires accurate estimation of the relative geometric relationship between the radar's flight path and the scene being imaged. Such geometry information is typically obtained from motion sensors such as inertial measurement units (IMUs) and global positioning system (GPS) receivers. These sensors, however, could be too expensive and may not provide the accuracy required for ultra-high resolution imaging. In addition, signal propagation through turbulent media is another critical factor that prohibits a SAR from achieving a high image resolution [1]. Consequently, signal based motion compensation, i.e., autofocus, is often an indispensable step that provides a necessary supplement to IMU/GPS devices, especially for airborne SAR processing, to achieve a very fine resolution.

A range measurement error imposes two adverse effects on the echoes, namely, azimuth phase error (APE) and residual range cell migration $(\mathrm{RCM})$. The APE makes an image defocused in the azimuth dimension, whereas the

This research is funded in part by the Doctoral Fund of Ministry of Education of China under contract no. 20123218120021, and the Fundamental Research Funds for the Central Universities under contract no. NS2012097. residual RCM introduces 2-D defocus. In the phase history domain, there exists a simple linear relationship between these two error terms. That is, the APE is the product of the residual RCM and a constant factor $4 \pi / \lambda$, where $\lambda$ is the wavelength. Therefore, for a SAR operated in a submeter wavelength $(4 \pi / \lambda \gg 1)$, the APE has a much more significant impact on the formed image than the residual RCM. When the range measurement error is small, e.g., within a range resolution cell, the effect of the residual RCM can be neglected and, as such, only the APE should be compensated. This is the general presumption made in most existing autofocus algorithms, such as Mapdrift (MD) [2], Phase Difference (PD) [3], Phase Gradient Autofocus (PGA) [4], and Eigenvector Method [5]. As the SAR resolution becomes finer, however, the increased synthetic aperture size makes the accumulated range measurement error more pronounced. On the other hand, range resolution cell becomes smaller. Therefore, the residual RCM will inevitably exceed the range resolution cell in future ultrahigh resolution SAR systems [6]. In this situation, 2-D autofocus becomes a necessary procedure to obtain refocused images.

Several 2-D autofocus methods have been proposed in literature (e.g., [7-10]). These techniques, however, perform phase error estimation in a blind manner because they do not take advantages of the known structure of the phase error. As such, they do not achieve accurate estimation of the 2-D phase error due to lack of data redundancy. Recently, Doerry et al. exploited the 2D phase error structure after some specific image formation algorithms, and proposed some preliminary knowledge aided 2D autofocus methods [11-13]. However, all these approaches simplified the 2D phase error into a combination of azimuth phase error and residual range cell migration.

In this paper, we provide further insights into the mathematical mechanism of the underlying SAR imaging in the polar format algorithm (PFA). Based on this understanding, we analyze the effect of range and azimuth resampling on the uncompensated phase error, and obtain the analytical structure of the residual 2-D phase error. These results are then exploited to develop a novel algorithm that achieves accurate 2-D autofocus. 


\section{POlar Format AlgOrithm}

Most efforts for PFA are focused on the implementation of the polar format transformation. The emphasis of this section rather lies in the exploitation of the analytical formulation in order to gain insightful observations into the mathematical mechanism within such transformations. The result will then be used in the next section for the analysis of phase errors.

\section{A. Signal Model}

Consider a spotlight-mode SAR operating with the geometry depicted by Fig.1. The coordinate of a generic stationary target in the illuminated scene in XOY is $\left(x_{\mathrm{p}}, y_{\mathrm{p}}\right)$. Let $t$ represent the slow time. The distance between the antenna phase center (APC) and the scene center (Point O) is $r_{\mathrm{c}} \equiv r_{c}(t)$, which along with the instantaneous squint angle $\theta \equiv \theta(t)$ and the incidence angle $\varphi \equiv \varphi(t)$ determines the instantaneous coordinate $\left(x_{a}, y_{a}, z_{a}\right) \equiv\left[x_{\mathrm{a}}(t), y_{\mathrm{a}}(t), z_{\mathrm{a}}(t)\right]$ of the APC. Denote $\theta_{\text {ref }}$ as the reference squint angle, and $\varphi_{\text {ref }}$ the corresponding incidence angle. For notational simplicity and without loss of generality, we define $t=0$ when $\theta=\theta_{\text {ref }}$.

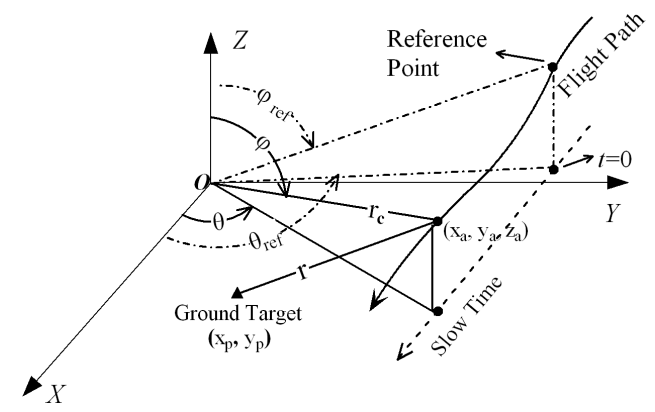

Figure 1. Spotlight SAR data collection geometry.

To proceed with the PFA, the radar echoes must be converted into the range-frequency domain. The conversion method depends on the type of operating modulations. If a linear frequency modulated (LFM) signal is transmitted, the deramp technique effectively transforms the echoes into the range-frequency domain at the receiver and reduces the required $\mathrm{A} / \mathrm{D}$ conversion rate. Alternatively, the conversion can be achieved by performing the Fourier transform to the sampled range signal. This approach is applicable to all modulation types. Without loss of generality, our discussion is based on the Fourier transform approach, whereas extension to the deramp method is straightforward.

After matched filtering and motion compensation with respect to the scene center, the 2-D signal can be expressed as

$$
S\left(f_{\mathrm{r}}, t\right)=A \cdot \exp \left\{\mathrm{j} \frac{4 \pi}{c}\left(f_{\mathrm{c}}+f_{\mathrm{r}}\right) \mathbf{R}_{\Delta}\right\}
$$

where $c$ is the speed of propagation, $f_{c}$ is the radar center frequency, $f_{\mathrm{r}}$ is the range frequency, $A$ includes the nonessential factors of the transmitted pulse envelop and azimuth antenna pattern, and

$$
\mathbf{R}_{\Delta}=r_{c}(t)-r(t)
$$

is the differential range, where $r(t)$ is the instantaneous distance between the APC and the target located at $\left(x_{\mathrm{p}}, y_{\mathrm{p}}\right)$.

The derivation of PFA is based on the planar wavefront approximation. Under this approximation, the differential range can be simplified as

$$
\mathbf{R}_{\Delta}=\sin \varphi\left(x_{\mathrm{p}} \cos \theta+y_{\mathrm{p}} \sin \theta\right)=\sin \varphi\left[\bar{x}_{\mathrm{p}} \sin \left(\theta_{\text {ref }}-\theta\right)+\bar{y}_{\mathrm{p}} \cos \left(\theta_{\text {ref }}-\theta\right)\right]
$$

where

$$
\left[\begin{array}{l}
\bar{x}_{\mathrm{p}} \\
\bar{y}_{\mathrm{p}}
\end{array}\right]=\left[\begin{array}{cc}
\sin \theta_{\text {ref }} & -\cos \theta_{\text {ref }} \\
\cos \theta_{\text {ref }} & \sin \theta_{\text {ref }}
\end{array}\right]\left[\begin{array}{l}
x_{\mathrm{p}} \\
y_{\mathrm{p}}
\end{array}\right] .
$$

By inserting (3) into (1), the 2-D signal can be rewritten as

$$
S\left(f_{\mathrm{r}}, t\right)=A \cdot \exp \left\{\mathrm{j} \frac{4 \pi}{c}\left(f_{\mathrm{c}}+f_{\mathrm{r}}\right) \sin \varphi\left[\bar{x}_{\mathrm{p}} \sin \left(\theta_{\text {ref }}-\theta\right)+\bar{y}_{\mathrm{p}} \cos \left(\theta_{\text {ref }}-\theta\right)\right]\right\} \text {. }
$$

Equation (5) shows that the phase history data are essentially a polar raster slice of the Fourier transform of the terrain reflectivity. Therefore, an image can be formed by applying the 2-D inverse discrete Fourier transform (IDFT). Unfortunately, efficient implementation of the transform through the 2-D fast Fourier transform (FFT) requires uniformly spaced samples on a rectangular grid. To exploit 2D FFT, therefore, the acquired polar samples of the phase history must be resampled to a rectangular grid. This is exactly what the PFA does. In order to reduce the computational burden, 2-D resampling can be decomposed into two tandem 1-D operations which, respectively, resample in range and azimuth. At the conclusion of these processes, the 2-D signal is reformulated with $f_{\mathrm{r}}$ and $t$ decoupled, and these two variables act as the respectively coefficients of $\bar{x}_{p}$ and $\bar{y}_{p}$.

From the point of view of range migration correction, the range migration is due to the coupling between range frequency and azimuth time in the phase history domain. As such, the polar reformatting can also be interpreted as a decoupling procedure.

\section{B. Range Resampling}

The range resampling is dedicated to eliminate the coupling between the range frequency and the azimuth time in the coefficient of $\bar{y}_{\mathrm{p}}$. This is achieved by transforming the coefficient of $\bar{y}_{\mathrm{p}}$ to be a linear function of the range frequency. Mathematically, this procedure is implemented by performing an azimuth time dependent change-of-variable on range frequency in (5), i.e., setting $f_{\mathrm{r}} \rightarrow \vartheta_{\mathrm{r}}\left(f_{\mathrm{r}} ; t\right)$. The objective of this change-of-variable is to achieve the following decoupling transformation:

$$
\left(f_{\mathrm{c}}+f_{\mathrm{r}}\right) \sin \varphi \cos \left(\theta_{\text {ref }}-\theta\right) \stackrel{f_{\mathrm{r}} \rightarrow v_{\mathrm{r}}\left(f_{\mathrm{r}} ; t\right)}{\longrightarrow}\left(f_{\mathrm{c}}+f_{\mathrm{r}}\right) \sin \varphi_{\text {ref }} .
$$

From (6), we can easily obtain 


$$
\vartheta_{\mathrm{r}}\left(f_{\mathrm{r}} ; t\right)=\delta_{\mathrm{r}} f_{\mathrm{r}}+f_{\mathrm{c}}\left(\delta_{\mathrm{r}}-1\right)
$$

where $\delta_{\mathrm{r}}=\sin \varphi_{\text {ref }} /\left[\sin \varphi \cos \left(\theta_{\text {ref }}-\theta\right)\right]$. It is clear from (7) that the range resampling, in essence, is a range frequency scaling transformation with a scaling factor of $\delta_{\mathrm{r}}$ and an offset of $f_{\mathrm{c}}\left(\delta_{\mathrm{r}}-1\right)$. This scaling transformation can be implemented through either interpolation or chirp scaling.

Undergoing the above change-of-variable, the echo signal becomes

$$
\begin{aligned}
& S_{\mathrm{R}}\left(f_{\mathrm{r}}, t\right)=S\left[\vartheta_{\mathrm{r}}\left(f_{\mathrm{r}} ; t\right), t\right] \\
& =A \cdot \exp \left\{\mathrm{j} \frac{4 \pi}{c}\left(f_{\mathrm{c}}+f_{\mathrm{r}}\right) \sin \varphi_{\text {ref }}\left[\bar{x}_{\mathrm{p}} \tan \left(\theta_{\text {ref }}-\theta\right)+\bar{y}_{\mathrm{p}}\right]\right\}
\end{aligned}
$$

It is evident from (8) that there is no longer a coupling between $f_{\mathrm{r}}$ and $t$ in the coefficient of $\bar{y}_{\mathrm{p}}$.

\section{Azimuth Resampling}

Similar to the range resampling, the azimuth resampling is dedicated to eliminate the coupling between the azimuth time and the range frequency in the coefficient of $\bar{x}_{p}$ in (8), i.e., make the coefficient only a linear function of the azimuth time. This procedure can be implemented by performing a range frequency dependent change-of-variable on the azimuth time, denoted as $t \rightarrow \vartheta_{\mathrm{a}}\left(t ; f_{\mathrm{r}}\right)$. This change-of-variable achieves the following transformation:

$$
\left(f_{\mathrm{c}}+f_{\mathrm{r}}\right) \tan \left(\theta_{\text {ref }}-\theta\right) \stackrel{t \rightarrow v_{\mathrm{a}}\left(t ; f_{\mathrm{r}}\right)}{\longrightarrow} f_{\mathrm{c}} \Omega t
$$

where $\Omega$ is a constant determined by this azimuth resampling process. If the APC travels ideally in parallel with OX (thus $\mathbf{y}_{\mathrm{a}}$ equals a constant $y_{0}$ ) at constant forward velocity $u_{\mathrm{x}}$, $\Omega$ can be typically set as $\Omega=u_{\mathrm{x}} \sin ^{2} \theta_{\text {ref }} / y_{0}$.

Without loss of generality, we consider that the radar platform may take an arbitrary path. In this case, the relationship between $\tan \left(\theta_{\text {ref }}-\theta\right)$ and $t$ can be described only numerically but not analytically. Therefore, it will be difficult to derive an analytical solution of $\vartheta_{\mathrm{a}}\left(t ; f_{\mathrm{r}}\right)$ from (9). To benefit our analysis in the next section, we divide the azimuth resampling, formulated as $\vartheta_{\mathrm{a}}\left(t ; f_{\mathrm{r}}\right)$, into two cascaded resampling procedures, which are expressed as $\vartheta_{\text {a1 }}(t)$ and $\vartheta_{\text {a2 }}\left(t ; f_{\mathrm{r}}\right)$, respectively. These two procedures are expressed as

$$
\left(f_{\mathrm{c}}+f_{\mathrm{r}}\right) \tan \left(\theta_{\mathrm{rff}}-\theta\right) \stackrel{t \rightarrow \vartheta_{\mathrm{a}}(t)}{\longrightarrow}\left(f_{\mathrm{c}}+f_{\mathrm{r}}\right) \Omega t \stackrel{t \rightarrow \rightarrow_{\mathrm{r} 2}\left(t ; f_{\mathrm{r}}\right)}{\longrightarrow} f_{\mathrm{c}} \Omega t .
$$

In the first procedure, a range frequency independent azimuth time transformation, i.e., $t \rightarrow \vartheta_{\text {a1 }}(t)$, is performed to linearize $\tan \left(\theta_{\text {ref }}-\theta\right)$. Such procedure is referred to as RCM linearization in this paper.
After the linearization process is performed, the $\tan \left(\theta_{\text {ref }}-\theta\right)$ term in (8) can be replaced by a linear function of $t$. That is, after RCM linearization, (8) becomes

$$
\begin{aligned}
& S_{\mathrm{L}}\left(f_{\mathrm{r}}, t\right)=S_{\mathrm{R}}\left[f_{\mathrm{r}}, \vartheta_{\mathrm{a} 1}(t)\right] \\
& =A \cdot \exp \left\{\mathrm{j} \frac{4 \pi}{c}\left(f_{\mathrm{c}}+f_{\mathrm{r}}\right) \sin \varphi_{\text {ref }}\left(\bar{x}_{\mathrm{p}} \Omega t+\bar{y}_{\mathrm{p}}\right)\right\}
\end{aligned}
$$

It is seen from (11) that the coefficient of $\bar{x}_{\mathrm{p}}$ is still range frequency dependent. That is, the decoupling is not completely achieved.

The second resampling procedure, formulated as $\vartheta_{\mathrm{a} 2}\left(t ; f_{\mathrm{r}}\right)$, is a range frequency dependent azimuth time resampling. From (10), we obtain

$$
\vartheta_{\mathrm{a} 2}\left(t ; f_{\mathrm{r}}\right)=\frac{f_{\mathrm{c}}}{f_{\mathrm{c}}+f_{\mathrm{r}}} t .
$$

This is the well-known Keystone transform (KT), which performs bulk correction of arbitrary linear RCM through the decoupling of the azimuth time and the range frequency.

After performing KT, the signal in (11) becomes

$S_{\mathrm{KT}}\left(f_{\mathrm{r}}, t\right)=S_{\mathrm{L}}\left[f_{\mathrm{r}}, \vartheta_{\mathrm{a} 2}\left(t ; f_{\mathrm{r}}\right)\right]=\tilde{A} \cdot \exp \left\{\mathrm{j} \frac{4 \pi}{c} \sin \varphi_{\mathrm{ref}}\left(\bar{x}_{\mathrm{p}} f_{\mathrm{c}} \Omega t+\bar{y}_{\mathrm{p}} f_{\mathrm{r}}\right)\right\}$

where $\tilde{A}=A \cdot \exp \left(\mathrm{j} \frac{4 \pi \sin \varphi_{\text {ref }}}{\lambda} \bar{y}_{\mathrm{p}}\right)$ is a constant.

\section{2-D Fourier Transform}

In (13), the azimuth time $t$ and the range frequency $f_{\mathrm{r}}$ are decoupled. The residual 2-D sinusoid can be converted into a focused target response via the 2-D Fourier transform with respect to $t$ and $f_{\mathrm{r}}$, resulting in the following product of two sinc functions

$$
\mathbf{F}\left[S_{\mathrm{KT}}\left(f_{\mathrm{r}}, t\right)\right]=\hat{A} \cdot \operatorname{sinc}_{\mathrm{r}}\left(\tau-\frac{2 \sin \varphi_{\text {ref }}}{c} \bar{y}_{\mathrm{p}}\right) \cdot \operatorname{sinc}_{\mathrm{a}}\left(f_{t}-\frac{2 \Omega \sin \varphi_{\text {ref }}}{\lambda} \bar{x}_{\mathrm{p}}\right)
$$

where $\mathbf{F}[\cdot]$ represents the 2-D Fourier transform, $f_{t}$ is the azimuth Doppler frequency, $\tau$ is the range time, $\operatorname{sinc}_{\mathrm{a}}(\cdot)$ and $\operatorname{sinc}_{\mathrm{r}}(\cdot)$ are azimuth and range sinc functions, respectively, which are defined by

$$
\begin{aligned}
& \operatorname{sinc}_{\mathrm{a}}(u)=\sin \left(\pi \mathrm{B}_{\mathrm{a}} u\right) /\left(\pi \mathrm{B}_{\mathrm{a}} u\right) \\
& \operatorname{sinc}_{\mathrm{r}}(u)=\sin \left(\pi \mathrm{B}_{\mathrm{r}} u\right) /\left(\pi \mathrm{B}_{\mathrm{r}} u\right)
\end{aligned}
$$

where $B_{a}$ and $B_{r}$ are the azimuth signal bandwidth and range signal bandwidth, respectively.

Equation (14) is the target's impulse response (IPR) function of the PFA with a far-field approximation. It becomes clear now that the azimuth resampling procedure in the PFA is, in essence, a combination of RCM linearization and KT. 
While an analytical expression of $\vartheta_{\text {a1 }}(t)$ may not always be available for an arbitrary radar flight path, this does not affect our analysis. We will show, in the next section, that only the KT contributes the derivation of analytical relationship between the APE and the residual RCM. It should be noted that, although the azimuth resample is divided into two individual procedures to facilitate our analysis, only one interpolation is required to accomplish both procedures of $\mathrm{RCM}$ linearization and $\mathrm{KT}$ in practical implementations of the PFA.

\section{RESIDUAL PHASE ERROR IN PFA IMAGE}

In the above development of PFA, we assumed that the relative range between the radar and the target scene is precisely known. In practice, however, range errors are usually inevitable due to inaccurate motion measurement or deleterious atmospheric effects. In this case, the actual differential range should be expressed as

$$
\mathbf{R}_{\Delta}=\sin \varphi\left[\bar{x}_{\mathrm{p}} \sin \left(\theta_{\text {ref }}-\theta\right)+\bar{y}_{\mathrm{p}} \cos \left(\theta_{\text {ref }}-\theta\right)\right]+\mathbf{R}_{\mathrm{E}}
$$

where $\mathbf{R}_{\mathrm{E}} \equiv R_{\mathrm{E}}(t)$ represents all the range errors.

Inserting (16) into (1), we can get the actual echo signal

$$
S\left(f_{\mathrm{r}}, t\right)=A \cdot \exp \left\{\mathrm{j} \frac{4 \pi}{c}\left(f_{\mathrm{c}}+f_{\mathrm{r}}\right)\left[\sin \varphi\left(\bar{x}_{\mathrm{p}} \sin \left(\theta_{\text {ref }}-\theta\right)+\bar{y}_{\mathrm{p}} \cos \left(\theta_{\text {ref }}-\theta\right)\right)+\mathbf{R}_{\mathrm{e}}\right]\right\} .
$$

From the previous section, we know that the range resampling in PFA is essentially a scaling transform in range frequency defined in (7). Therefore, after this range resampling, the signal in (17) becomes

$$
\begin{aligned}
& S_{\mathrm{R}}\left(f_{\mathrm{r}}, t\right)=S\left[\vartheta_{\mathrm{r}}\left(f_{\mathrm{r}} ; t\right), t\right] \\
& =A \cdot \exp \left\{\mathrm{j} \frac{4 \pi\left(f_{\mathrm{c}}+f_{\mathrm{r}}\right) \sin \varphi_{\text {ref }}}{c}\left[\bar{x}_{\mathrm{p}} \tan \left(\theta_{\text {ref }}-\theta\right)+\bar{y}_{\mathrm{p}}+\varepsilon\right]\right\}
\end{aligned}
$$

where $\varepsilon \equiv \varepsilon(t)=\mathbf{R}_{\mathrm{E}} /\left[\sin \varphi \cos \left(\theta_{\text {ref }}-\theta\right)\right]$.

The second step of PFA is azimuth resampling. To benefit the following analysis, we have divided it into two cascaded resampling procedure, i.e., RCM linearization and KT. After $\mathrm{RCM}$ linearization, the signal in (18) becomes

$$
\begin{aligned}
& S_{\mathrm{L}}\left(f_{\mathrm{r}}, t\right)=S_{\mathrm{R}}\left[f_{\mathrm{r}}, \vartheta_{\mathrm{a} 1}(t)\right] \\
& =A \cdot \exp \left\{\mathrm{j} \frac{4 \pi}{c}\left(f_{\mathrm{c}}+f_{\mathrm{r}}\right) \sin \varphi_{\text {ref }}\left(\bar{x}_{\mathrm{p}} \Omega t+\bar{y}_{\mathrm{p}}+\eta\right)\right\}
\end{aligned}
$$

where $\eta \equiv \eta(t)=\varepsilon\left[\vartheta_{\text {a1 }}(t)\right]$.

The final step is to perform KT on (19), which results in

$$
\begin{aligned}
& S_{\mathrm{KT}}\left(f_{\mathrm{r}}, t\right)=S_{\mathrm{L}}\left[f_{\mathrm{r}}, \vartheta_{\mathrm{a} 2}\left(t ; f_{\mathrm{r}}\right)\right] \\
& =\tilde{A} \cdot \exp \left\{\mathrm{j} \frac{4 \pi}{c} \sin \varphi_{\text {ref }}\left(\bar{x}_{\mathrm{p}} f_{\mathrm{c}} \Omega t+\bar{y}_{\mathrm{p}} f_{\mathrm{r}}\right)+\phi\left(f_{\mathrm{r}}, t\right)\right\}
\end{aligned}
$$

where $\tilde{A}=A \cdot \exp \left(\mathrm{j} \frac{4 \pi \sin \varphi_{\text {ref }}}{\lambda} \bar{y}_{\mathrm{p}}\right)$ is a constant, and

$$
\phi\left(f_{\mathrm{r}}, t\right)=\frac{4 \pi\left(f_{\mathrm{c}}+f_{\mathrm{r}}\right) \sin \varphi_{\mathrm{ref}}}{c} \eta\left(\frac{f_{\mathrm{c}}}{f_{\mathrm{c}}+f_{\mathrm{r}}} t\right)
$$

is the 2-D phase error.

From (21), we observe that the 2-D phase error possesses a fine internal structure whose parameters, except $\eta(t)$, are known a priori. Therefore, only $\eta(t)$ needs to be evaluated to obtain the 2-D phase error.

In practice, $\eta(t)$ can be evaluated from the APE. By setting $f_{\mathrm{r}}=0$ in (21), the APE is obtained as

$$
\phi_{0}(t)=\frac{4 \pi f_{0} \sin \varphi_{\mathrm{ref}}}{c} \eta(t) .
$$

It is clear from (21) and (22) that there exists a mapping between the 2-D phase error and the azimuth 1-D phase error, expressed as

$$
\phi\left(f_{\mathrm{r}}, t\right)=\frac{f_{0}+f_{\mathrm{r}}}{f_{0}} \phi_{0}\left(\frac{f_{0}}{f_{0}+f_{\mathrm{r}}} t\right) .
$$

Therefore, the 2-D phase error can be computed directly from the azimuth 1-D phase error based on this formula.

\section{KNOWLEDGE-AIDED 2-D AutoFocus}

Conventional 2-D autofocus algorithms assume no knowledge of the 2-D phase error in the estimation of phase error. Such methods, which are referred to as blind estimation techniques of the phase error, have at least two drawbacks in actual applications. First, there is not enough data redundancy that can be used to improve the estimation accuracy of the phase error. The conventional 1-D estimation of the phase error exploits the data redundancy in the range dimension. As the parameter space changes from 1-D to 2-D, however, the data redundancy significantly decreases. As a result, the performance of the 2-D phase error estimation can hardly be assured. Second, compared with the 1-D phase error estimation, the computation complexity of the 2-D phase error estimation increases exponentially, rendering real-time processing infeasible or difficult.

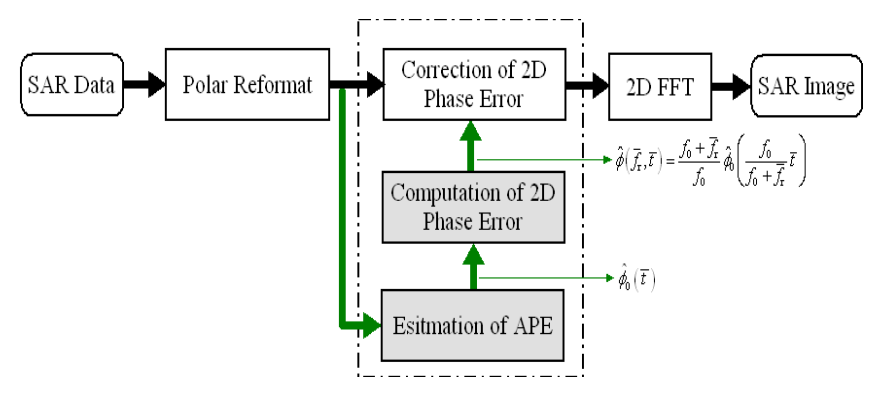

Figure 2. Flow of 2-D autofocus processing 
As we discussed earlier, partial information of the residual 2-D phase error is available and there exists a specific internal structure. By exploiting this structure, the 2-D phase error estimation problem can be simplified as a 1-D estimation problem of the APE. Based on this idea, we propose a 2-D autofocus algorithm based on 1-D estimation and 2-D compensation, as shown in Fig. 2. The key to this algorithm consists of two parts: estimation of the APE and computation of the 2-D phase error.

\section{A. Azimuth Phase Error Estimation}

The APE estimation can be accomplished by conventional autofocus algorithms. If these algorithms are exploited without proper modifications, however, the phase error estimation performance degrades in the case that the residual range migration cannot be ignored. A straightforward method to resolve this problem is to reduce the range resolution prior to the estimation of the phase error. As such, the residual range migration is confined into one range resolution cell. The APE is then estimated using conventional autofocus algorithms.

\section{B. Computation of 2-D Phase Error}

According to (23), we can see that the mapping from APE to the 2-D phase error is one-to-one and flight-path independent. Thereby, once the APE is estimated, the 2-D phase error can be calculated directly. This direct computation of 2-D phase error eliminates a 2-D blind estimation process, thereby possessing a high computational efficiency.

\section{EXPERIMENTAL RESULTS}

Real data collected by an experimental airborne SAR is applied to demonstrate the effectiveness of the proposed autofocus methods. The experimental radar operates in spotlight mode at X-band. Its transmitted signal has a bandwidth of $1.18 \mathrm{GHz}$, corresponding to $0.13 \mathrm{~m}$ theoretical range resolution. The processed synthetic aperture length is about $2300 \mathrm{~m}$, hence the nominal azimuth resolution would be $0.067 \mathrm{~m}$. Because no motion sensor data is available in hand, we assume that the radar platform flies at a constant velocity in image formation. Furthermore, an artificially velocity bias is used to make the 2-D defocus more clearly. Fig.3 (a) is the image produced by PFA processing without any autofocus procedure applied. Although deterministic range migration has been compensated by polar format transformation, residual RCM is still large enough to exceed several range resolution cells. It can be clearly seen that the image suffers from severely 2-D defocus, i.e., the image exhibits smearing not only in the azimuth direction, but also in the range direction due to residual RCM. In this situation, conventional autofocus algorithm can't completely compensate for the errors. Residual degradation in image is still substantial. This can be clearly seen in Fig.3(b), which is produced by PFA with PGA applied. Fig.3(c) is the result using our method. It is clear that the reconstructions exhibit excellent quality.

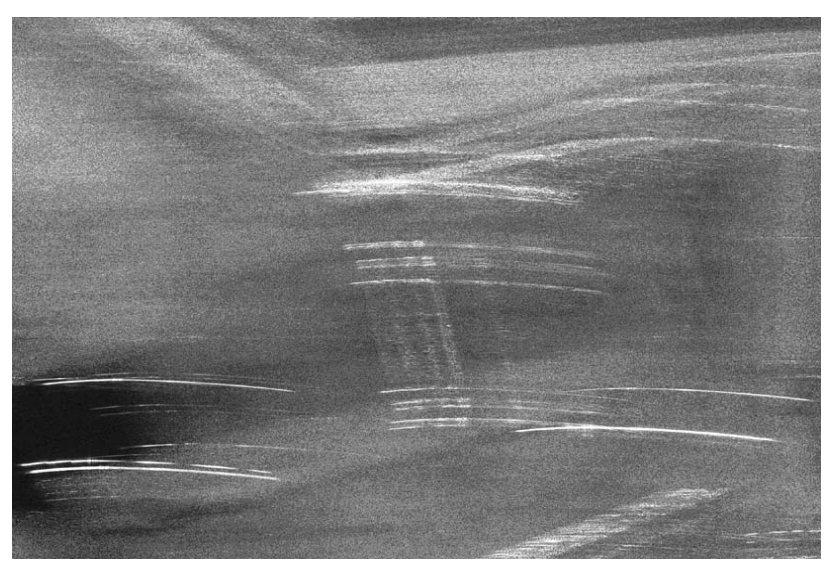

(a) PFA image

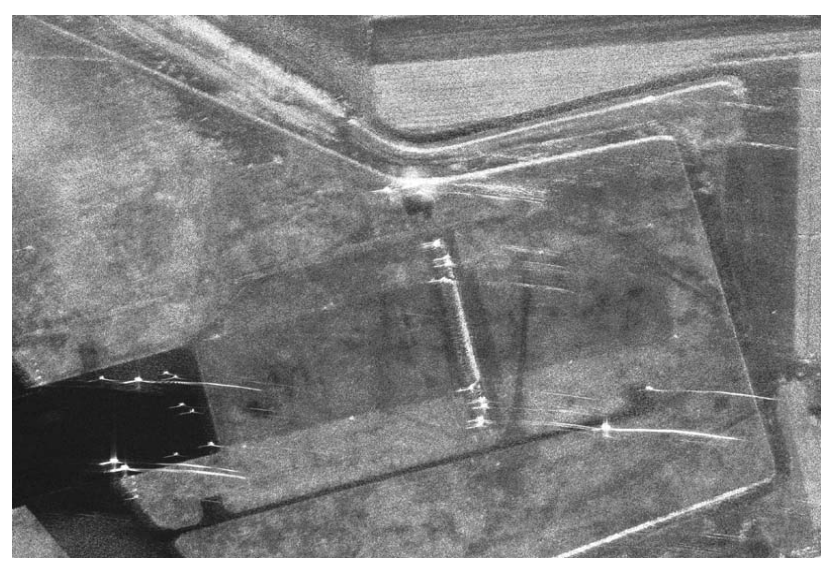

(b) Refocused by PGA

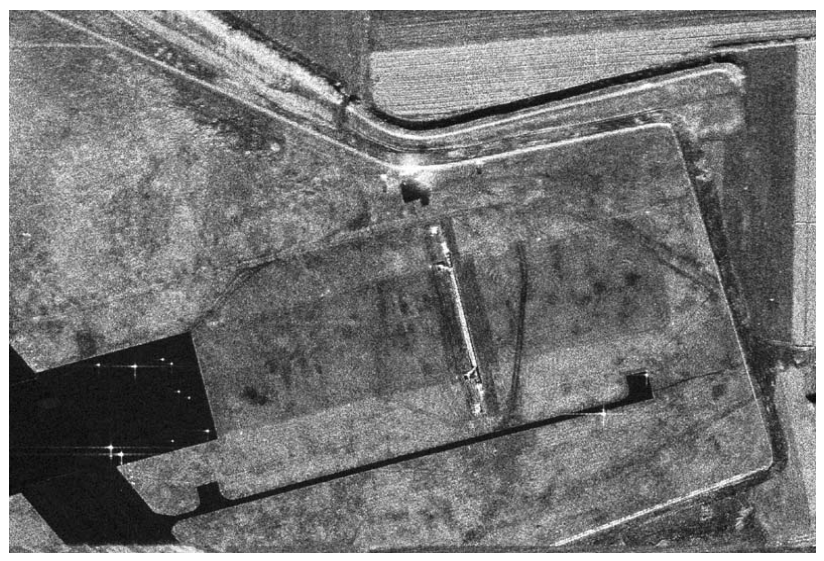

(c) Refocused by the proposed 2D autofocus method

Figure 3. Experimental results

\section{CONCLUSION}

This paper has analyzed in detail the analytical structure of the residual 2-D phase error after the imaging processing and changed the problem of the 2-D phase error estimation into that of 1-D phase error estimation with exploitation of this priori aided knowledge. Then an autofocus algorithm based on 
this 1-D estimation/2-D correction is proposed. This algorithm needs only to directly estimate the 1-D azimuth phase error and maps this 1-D phase error into $2-\mathrm{D}$ phase error with the use of the specific structure information inside the phase error. Thus the accurate estimation and compensation of the 2-D phase error are accomplished. This new approach not only decreases the computation complexity, but also solves the problem that the phase cannot be accurately estimated due to lack of data redundancy by the conventional 2-D autofocus algorithms.

\section{REFERENCES}

[1] M. Denny and I. Scott, "Anomalous propagation limitations to high-resolution SAR performance," Proceedings of the IEEE Radar Conference, Long Beach, CA, pp.249-254, 2002.

[2] C. E. Mancill and J. M. Swiger, "A map drift autofocus technique for correcting high order SAR phase error," Record of the 27th Annual Tri-Service Radar Symposium, Monterey, CA, pp. 391-400, 1981.

[3] G. N. Yoji, "Phase difference auto focusing for synthetic aperture radar imaging," United States Patent, no. 4999635, 1991.

[4] D. E. Wahl, P. H. Eichel, D. C. Ghiglia, and C. V. Jakowatz, "Phase gradient autofocus -- A robust tool for high resolution SAR phase correction," IEEE Transactions on Aerospace and Electronic Systems, vol. 30, no. 3, pp. 827-834, 1994.

[5] C. V. Jakowatz and D. E. Wahl, "Eigenvector method for maximum-likelihood estimation of phase error in synthetic aperture radar imagery," Journal of the Optocal Society of
America A, vol. 10, no. 12, pp. 2539-2546, 1993.

[6] D. Kirk and P. Maloney, "A new autofocus technique for wideband wide-angle synthetic aperture radar," Proceedings of SPIE, vol. 3370, pp.52-61, 1998.

[7] D. W. Warner, D. C. Ghiglia, A. FitzGerrel, and J. Beaver, "Two-dimensional phase gradient autofocus," Proceedings of SPIE, vol. 4123, pp.162-173, 2000.

[8] A.Gallon and F. Impagnatiello, "Motion compensation in chirp scaling SAR processing using phase gradient autofocusing," Proceedings of IEEE Geoscience and Remote Sensing Symposium, vol. 2, pp. 633-635, 1998.

[9] D. Zhu, "SAR signal based motion compensation through combining PGA and 2-D map drift," Proceeding of 2nd AsianPacific Conference on Synthetic Aperture Radar, pp. 435-438, 2009.

[10] J. C. Kirk, R. Lefevre, R. Van Dalen Wetters, D. Woods, and B. Sullivan, "Signal based motion compensation (SBMC)," Proceedings of the IEEE International Radar Conference, pp. 463-568, 2000.

[11] A.W. Doerry, "Autofocus correction of excessive migration in synthetic aperture radar images," Sandia Report, SAND20044770, September 2004.

[12] L. Yang, M. Xing, et al., "Compensation for the NsRCM and phase error after polar format resampling for airborne spotlight sar raw data of high resolution," IEEE Geoscience and Remote Sensing Letters, vol.10, no.1, pp.165-169, 2013.

[13] X. Mao, D. Zhu, et al., "Autofocus correction of azimuth phase error and residual range cell migration in spotlight SAR polar format imagery," IEEE Transactions on Aerospace and Electronic Systems, in press. 